\title{
Erratum: Correspondence: Reply to 'Enhancing a phase measurement by sequentially probing a solid-state system'
}

Liu Gang-Qin, Zhang Yu-Ran, Chang Yan-Chun, Yue Jie-Dong, Fan Heng \& Pan Xin-Yu

Nature Communications 7:11521 doi: 10.1038/ncomms11521 (2016); Published 9 May 2016; Updated 7 Jun 2016

The HTML version of this Correspondence was previously published with an incomplete title. This has now been corrected in the HTML; the PDF version of the paper was correct from the time of publication. 